\title{
45 Jahre Ultraschallprüfung in der Schweiz
}

Die langjährige und vielseitige Tradition der Ultraschallprüfung hat sich in der Schweiz vor allem im Maschinenbau entwickelt. Der Beitrag zeigt die entscheidenden Stadien von den Anfängen der Handprïfung bis zur rechnerintegrierten Prüftechnik chronologisch auf. Dabei veranschaulichen einige Beispiele diese Entwicklung.

\section{Erste Ultraschalluntersuchungen an Schmiedeteilen}

Der Bau von zunehmend größeren Maschinen in den vierziger Jahren und die dabei auftretenden zahlreichen Schadensfälle von Schmiedeteilen wie Rotoren und Kurbelwellen machten die Entwicklung von Prüfmethoden zum frühzeitigen Entdecken von Fehlern notwendig. Die Magnet-Rißprüfung als OberflächenPrüfverfahren konnte den Anforderungen nicht genügen, lagen doch die größten Fehler wie Lunker oder Einschlüsse im Inneren der Schmiedestücke.

Die Einführung von Sonar-Geräten eröffnete neue Prüfmöglichkeiten. Ein englisches Ultraschallgerät Kelvin-Hughes wird erstmals 1946 in einem Prüfattest erwähnt [1]. Die damalige Gerätetechnik erlaubte nur getrennte Sender und Empfänger. Um einen guten Kontakt der Prüfköpfe zu gewährleisten und Sendeund Empfangsebene zur Deckung zu bringen. wurden z.B. an Rotorwellen vier um $90^{\circ}$ versetzte Flachbahnen von $30 \mathrm{~mm}$ Breite in Längsrichtung gefräst (Bild 1). Diese Prüfbahnen wurden sauber geschliffen, um sicherzustellen, daß die geringe Sendeenergie gut in den Prüfling gelangt. Die senkrechte Durchschallung erfolgte ausschließlich entlang dieser vier Prüfbahnen. Als Justierkörper diente ein Stahlblock mit Zylinderbohrungen. Gesucht wurden hauptsächlich große Lunker, Einschlüsse und Risse. Die Ultraschallanzeigen hatten eine kleine Amplitude und die zeitliche Auflösung war beschränkt. Eine solche Anzeige in $206 \mathrm{~mm}$ Tiefe und den entsprechenden metallographischen Schnitt zeigt Bild 2. Es handelt sich dabei um einen Schmiederiß durch lokale Sulfidanreicherung in einer Welle von $500 \mathrm{~mm}$ Durchmesser.

\section{Schweißnahtprüfung und Druckleitungsbau}

Beim Ausbau der Wasserkraft waren die Hersteller von Druckleitungen auf eine empfindliche Methode zur zerstörungsfreien Prüfung der Schweißnähte angewiesen. Die Durchstrahlungsprüfung auf Baustellen an den Steilhängen der Berge und in den Stollen war problematisch und wie sich herausstellte, nicht sehr zuverlässig. Nach der erfolgreichen Anwendung der Ultraschallprüfung an Schmiedestücken lag es nahe, diese Technik auch an Schweißverbindungen anzuwenden. Ein Ultraschallgerät mit getrenntem Sender und Empfänger eignete sich nicht für diese Aufgabe. Erst das Ultraschallgerät USIP6 der Krautkrämer GmbH, Hürth, machte 1953 mit dem Sender/EmpfängerMonokopfsystem die Prüfung von Schweißnähten auf der Baustelle handhabbar.

Erste Versuche mit Winkelprüfköpfen für die Schweißnahtprüfung wurden bereits im April des gleichen Jahres durchge-

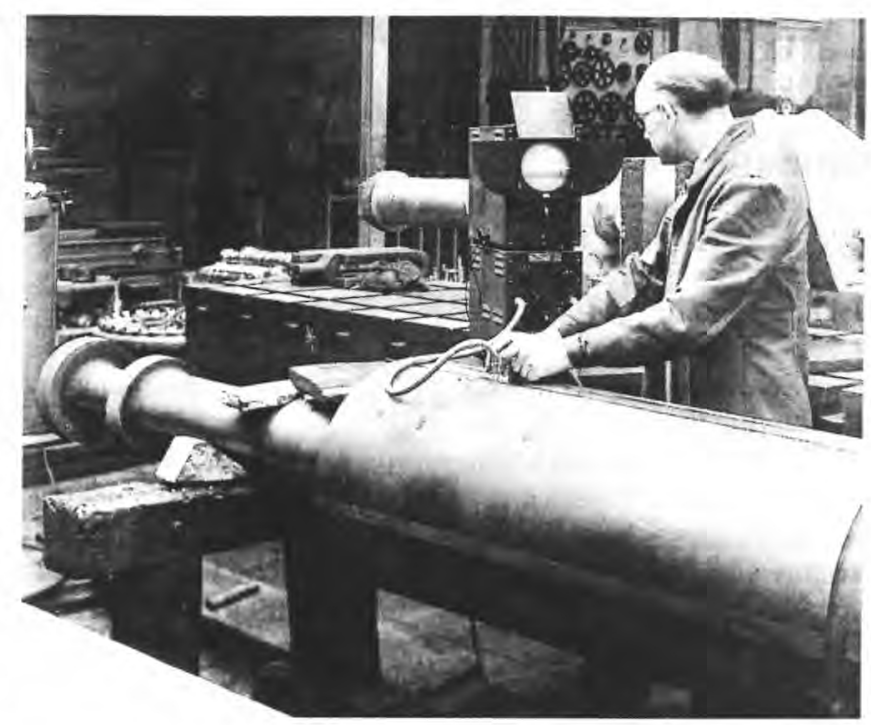

Bild I. Priifung eines Gasturbinen-Rotors entlang vier Flachbahnen

führt [2]. Die Sendeimpulsstärke und die Empfangsverstärkung wurde nach Erfahrung eingestellt. Eine Justierung der Empfindlichkeit an Testreflektoren fand noch nicht statt. Zur Interpretation der Anzeigen wurde festgestellt, daß „,kleine Risse sauberer Trennung schon sehr große Fehlerechos hervorrufen, dagegen Poren und Schlacken von größerem Ausmaß keine hohen Fehleranzeigen aufweisen, jedoch die Fehlerortungen bequem sind, da sie meist nur sehr lokal sind und unter verschiedenen Winkeln maximale Fehleranzeigen aufweisen" [2].

Die Lokalisierung der Fehlerstellen in dicken Stücken war dagegen nicht möglich. Dies lag an der falschen Annahme, daß die Geschwindigkeit der Transversalwellen exakt gleich der Hälfte der Longitudinalwellen war. Die Justierung der Zeitablenkung mit einem Normalprüfkopf und die Korrektur um einen Faktor zwei am Gerät führte zwangsläufig bei großen Schallwegen zu merkbaren Abweichungen. Weitere Schwierigkeiten entstanden bei der iterativen Korrektur der Vorlaufstrecke anhand der Beschallung zweier Zylinderbohrungen.

Die Entwicklung eines Testkörpers im Jahre 1954 [3] ermöglichte zum ersten Mal die richtige Einstellung der Transversalwellen-Geschwindigkeit. Die beiden Radien von 75 und $140 \mathrm{~mm}$

45 years of ultrasonic testing in Switzerland. The long and versatile tradition of ultrasonic testing in Switzerland has developed mainly in machine building industry. Starting with a Kelvin Hughes instrument in 1946 the development went through all stages from hand held testing to automatived and computer integrated testing. A wide range of applications including machine shajis, pressure pipes, austenitic welds and coatings are reported. 


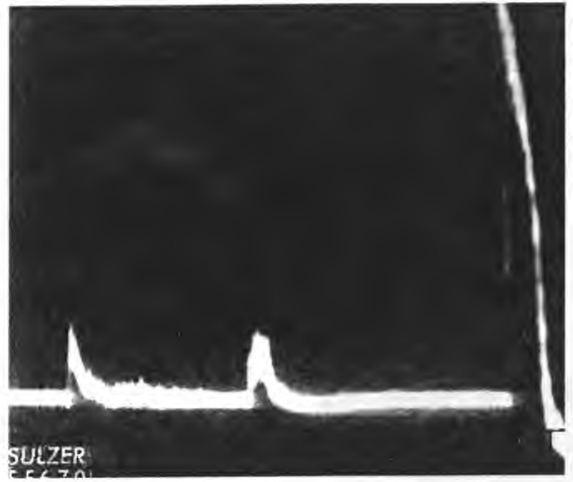

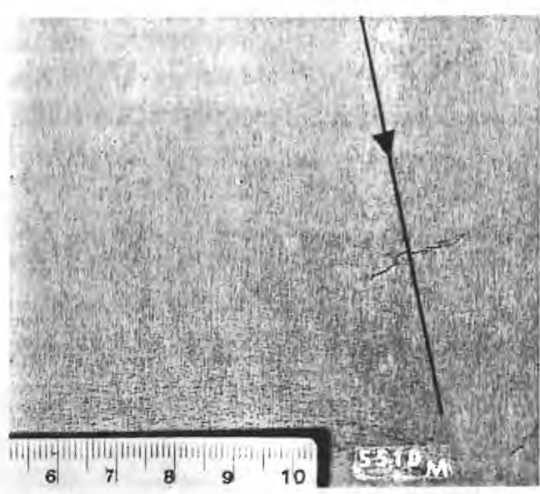

Bild 2. Anzeige eines Schmiederisses kurz vor Wellenmitte (links) mit metallographischem Schnitt (rechts) erlaubten zusätzlich die Bestimmung des Schallaustrittspunkts entsprechend des Zentrums der gemeinsamen Kreisbögen, ebenso die Ermittlung des Einschallwinkels bei indirekter Einschallung von der Rückseite des Testkörpers an einer Skala. Einige Jahre später ermöglichten eingebrachte Zylinderbohrungen von $1,2 \mathrm{~mm}$ Durchmesser für die Winkel $45^{\circ}, 60^{\circ}$ und $70^{\circ}$ sowie einem Schallweg von $60 \mathrm{~mm}$ die reproduzierbare Einstellung der Prüfempfindlichkeit.

Die Entwicklung von Lokalisationsskalen für diese häufig benutzten Einschallwinkel ermöglichte die Ortung der Fehler und war damit ein wichtiges Hilfsmittel für die Interpretation der Anzeigen. Die erste Version basierte auf dem Abstand des Schall-
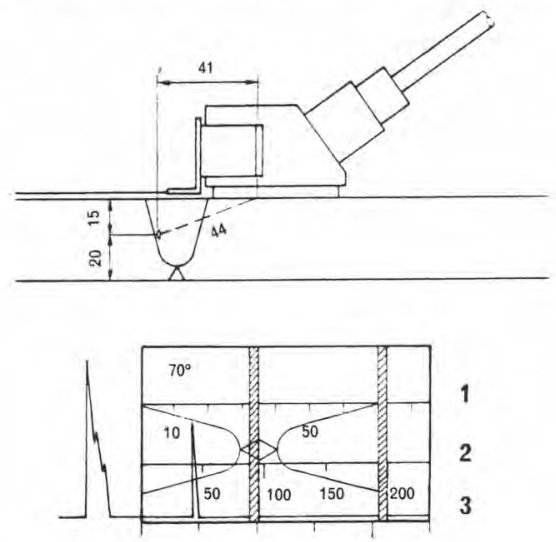

Bild 3. Lokalisations-Skala für die Fehlerortung unter diskreten Einschallwinkeln

austrittspunkts von der Nahtmitte und ermittelte auf komplizierte Art die Lage des Fehlers relativ zur Nahtmitte. Die spätere Version von 1956 stellte die trigonometrischen Relationen zwischen Schallweg, Projektionsabstand und Tiefe graphisch auf einem Vorsatzschirm dar, auf dem die drei zugehörigen Skalen horizontal eingetragen waren - eine große Hilfe für den Prüfer (Bild 3).

Im April 1954 wurden mit großem Erfolg sämtliche Montagenähte der Druckleitung Mauvoisin zu $100 \%$ ultraschallgeprüft, während die vorgesehene Durchstrahlungsprüfung mit Isotopen schon bei der Prüfung der ersten Verbindungen versagt hatte. Seit diesem Zeitpunkt wurde die Ultraschallprüfung als Ersatz der Röntgenprüfung eingeführt. Die Durchstrahlungsprüfung wurde nur noch auf ausdrücklichen Wunsch des Kunden bzw. der Abnahmegesellschaft durchgeführt.

\section{Austenitische Werkstoffe}

Schweißverbindungen austenitischer Rohrleitungen stellten andere Anforderungen an die Ultraschallprüfung als ferritische.
Einige Komponenten für den Primärkreislauf von Kernkraftwerken der ersten Generation in den sechziger Jahren, Beznau I und II sowie Mühleberg, waren aus austenitischem Stahl, dessen Anisotropie, Grobkörnigkeit des Gefüges und Inhomogenität der Schweißverbindungen zu Absorption, Streuung und Wellenumwandlungen führte. Die Anzeigen von Fehlern waren von Scheinanzeigen und Gefügerauschen überdeckt und ließen sich nicht oder nur schwer identifizieren. Eine merkliche Verbesserung brachte die Verwendung breitbandiger, fokussierter und an die Rohrkrümmung angepaßter SEL-Winkelprüfköpfe niedriger Frequenz.

\subsection{Fertigungsprïfung}

Die ersten erfolgreichen Schritte der Prüfung von austenitischen Komponenten waren mit dem Bau der Kernkraftwerke der zweiten Generation. Gösgen und Leibstadt, verbunden [4]. Gefügerauschen und Schallschwächung des grobkörnigen Werkstoffs sind an einem Nimonic Stück beeindruckend dargestellt (Bild 4). Die Prüfung einer Mischnaht eines Safe-Ends mit $45^{\circ}$ Longitudinalwellen führte zu einer teilweisen Ablenkung der Schallwellen am Übergang zwischen 18.8 Grundmaterial und Inconel Schweißgut. Diese trafen die Rückwand fast senkrecht und führten zu einer starken Scheinanzeige (Bild 5). Das Signal verschwand abrupt, sobald der Schallaustrittspunkt des Prüfkopfs im Schweißnahtbereich lag.

Für die Ultraschallprüfung von austenitischen Schweißverbindungen wurde es übliche Praxis, die Amplitude einer Anzeige als Differenz zur Amplitude eines Testreflektors im entsprechenden Vergleichskörper anzugeben. Bei einer manuellen Prüfung ist ein solcher Wert nicht sehr anschaulich. Konkreter (aber auch irreführend) wird die Ersatzreflektorgröße (ERG) verwendet, der Durchmesser jenes Kreisscheibenreflektors, der im AVG-Bewertungssystem die gleiche Amplitude hätte. Einen solchen Testreflektor für jeden Einschallwinkel herzustellen, ist praktisch nicht möglich und extrem aufwendig.

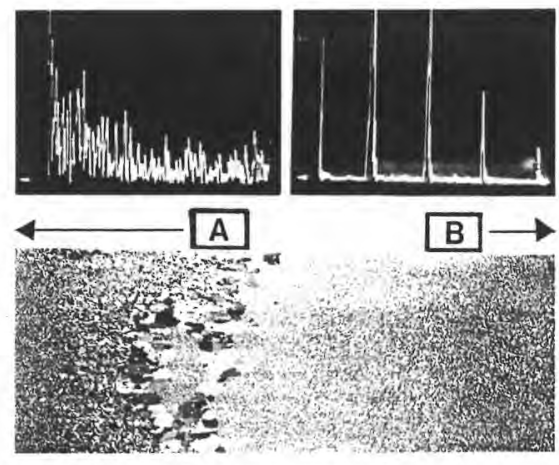

Bild t. Einfluß der Grobkörnigkeit und der Schallschwächung: Gefïgeanzeigen bei $A$, Riickwandechofolge bei $B$ 


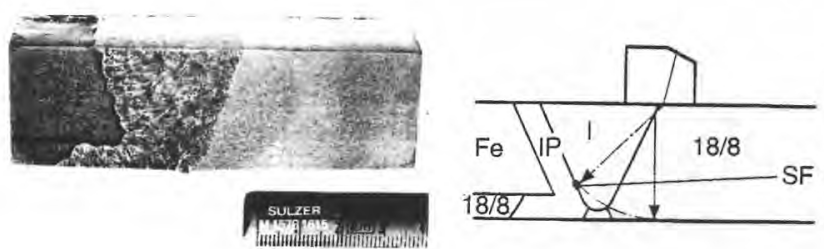

Bild 5. Scheinanzeige durch Ablenkung an der Nahtflanke; IP InconelPufferschweißung, I Inconel; SF scheinbarer Fehler

Daher werden als Reflektoren im Inneren von Testkörpern meist Zylinderbohrungen verwendet. Sie eignen sich dank ihrer Richtcharakteristik für alle Einschallwinkel. Der Übergang von einem Reflektortyp zum anderen kann mittels einer Umrechnungsformel hergestellt werden und ist z.B. im KTA-Regelwerk angegeben. Als Erleichterung für den Prüfer wird diese Relation zwischen Zylinderbohrung und Kreisscheibenreflektor in einem Umrechnungsschieber berücksichtigt (Bild 6) [5]. Die Arbeiten zur Fehlerbewertung mit Ultraschall sowie die Ultraschallprüfung von austenitischen Schweißverbindungen fanden auch international Anerkennung [6].

\subsection{Wiederkehrende Prüfung}

Basierend auf den Erfahrungen mit der Ultraschallprüfung von austenitischen Schweißverbindungen in der Fertigung wurden für das Kernkraftwerk Beznau von 1978 bis 1980 Entwicklungsarbeiten und wiederkehrende Prüfungen an austenitischen

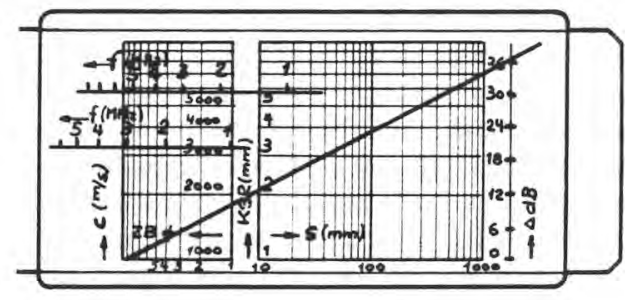

Bild 6. Schieber für die Umrechnung der Anzeigen von Zylinderbohrungen in äquivalente Kreisscheibenreflektoren

Primärkreislauf-Leitungen mit komplizierten Safe-End-Geometrien in Verbindung mit austenitischem Guß durchgeführt [7, 8]. Die manuellen Prüfungen wurden an einer ausgewählten Anzahl von Nähten durchgeführt und die Resultate sehr sorgfältig dokumentiert.

Die wachsenden Anforderungen bezüglich Empfindlichkeit, Reproduzierbarkeit und Dokumentation konnten nur mit einer mechanisierten Prüfung erfüllt werden. Deshalb wurde 1983 an der Treibwasserschleife des Kernkraftwerks Würgassen das erste mal teilmechanisiert geprüft [9]. Der Prüfer bewegte den Prüfkopf, sorgte für die Ankopplung mit Koppelpaste und tastete die Naht in kleinen Abschnitten mit zwei Einschallrichtungen ab. Das System ordnete aus der Lage des Schallaustrittspunkts, der Einschallrichtung und der Laufzeit die Anzeigen einem Volumenelement des Nahtbereichs zu. Wegen der notwendigen Reduktion der Datenmenge wurden anstelle der Volumeninformation die drei Projektionen abgespeichert und konnten so nachträglich ausgedruckt und ausgewertet werden. Schon ein Jahr später wurde im Kernkraftwerk Mühleberg mit einem vollmechanisierten Manipulator sehr zuverlässig gearbeitet.

An Siedewasserreaktoren von General Electric sind zwischen 1982 und 1985 gehäuft Fälle von interkristalliner Spannungsrißkorrosion (IKSRK) an austenitischen Rohrleitungen entdeckt worden, was insbesondere in USA zu ausgedehnten Reparatu- ren geführt hat. Aus diesem Grund wurde bereits 1979 mit manuellen Ultraschallprüfungen an der austenitischen Umwälzschleife im Kernkraftwerk Mühleberg begonnen. An vorhandenen Probestücken und Rohrausschnitten mit echten und künstlich erzeugten Rissen und Spannungsrißkorrosion wurde die Untersuchungstechnik entwickelt. Im Hinblick auf nur einseitig prüfbare Schweißnähte war der Fehlernachweis z.T. durch die Schweißnähte hindurch zu führen.

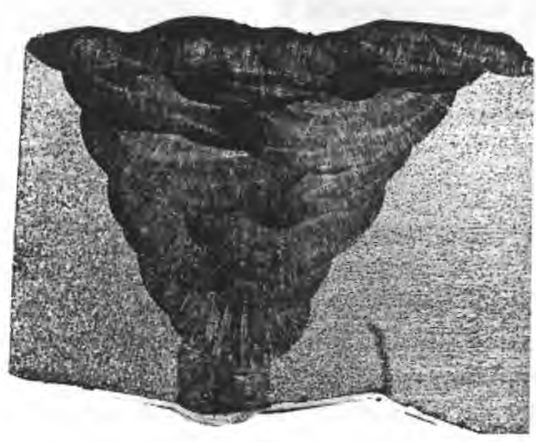

Bild 7. Interkristalline Spannungsriß-Korrosion in einer austenitischen $22 \mathrm{~mm}$-Schweißnaht

Die ersten Prüfungen wurden als Handprüfungen durchgeführt. Dieses Vorgehen war gerechtfertigt durch die wenigen, teils nur lokalen Prüfungen. Im Jahre 1983 stellte man manuell größere Anzeigenveränderungen fest. 1984 wurde beschlossen, den Prüfumfang auszuweiten. Die Anzahl der Schweißnähte ließ eine Handprüfung aus Gründen der Strahlenbelastung für das Prüfpersonal nicht zu. Der Einsatz von automatisierten Prüf- und Registriersystemen drängte sich daher auf [10]. Eine 1984 herausgetrennte Naht $43 \mathrm{~F}$ wurde mit der Farbeindringprüfung und metallurgisch untersucht. Der US-Befund der fast umlaufenden
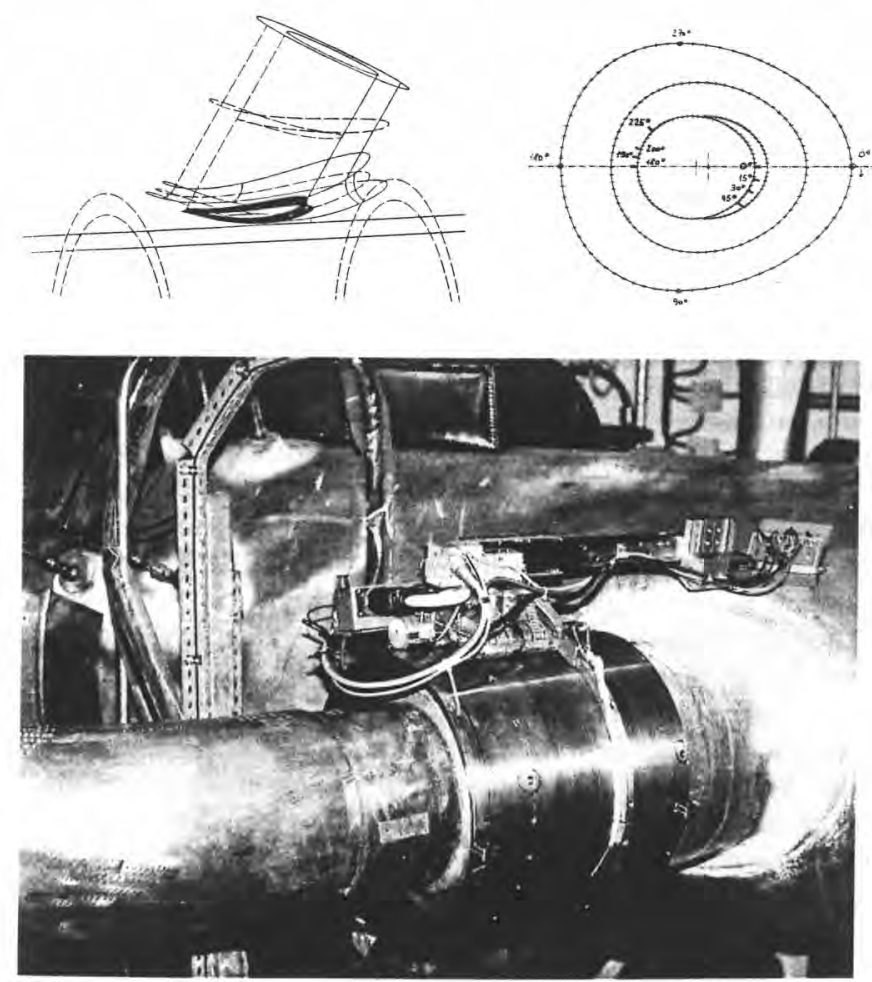

Bild 8. Prüfung der Stutzeninnenkante: Priifsituation vor Ort (unten), CAD-Darstellung des Prüfbereichs. Fahrkurven mit eingezeichneter Nutenlage (oben) 


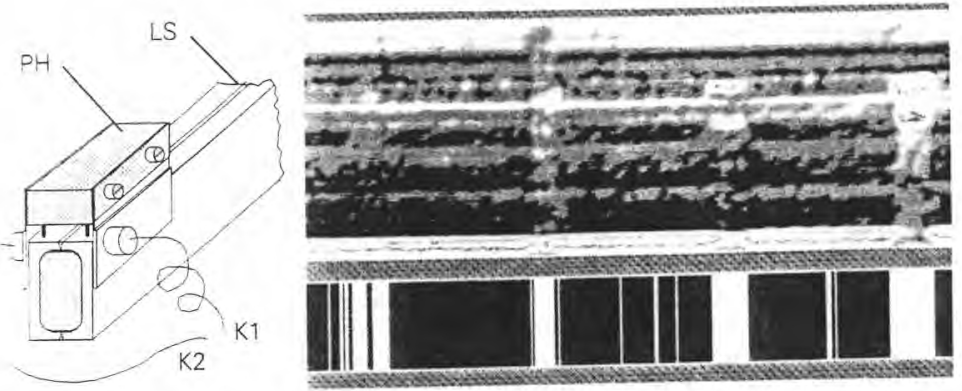

Bild 9. Prüfanordnung (links) von Laserschweißungen mit automatischer Signalerkennung(rechts): LS Laserschweißung; PS Prïfkopfhalterung und-führung; K1,K2 separate Meßkanäle

Spannungsrißkorrosion wurde bestätigt. Interkristalline Spannungsrisse ließen sich sowohl an der Nahtflanke als auch von der Andrehung ausgehend nachweisen (Bild 7). 17 weitere austenitische Schweißnähte wurden mechanisiert mit P-Scan geprüft. 1985 wurde der mechanisierte Prüfumfang auf 30 Nähte ausgeweitet. Zusätzlich wurden rund 40 weitere Nähte manuell überprüft.

Aufgrund dieser Prüfergebnisse wurde schon 1984 entschieden, die Umwälzschleife zu ersetzen. Bei der Austauschaktion im Jahre 1986 wurde während der Montage die mechanisierte Fertigungsprüfung sämtlicher Nähte durchgeführt. Diese Prüfung galt gleichzeitig als Basismessung für spätere Wiederholungsprüfungen. Um mit der Fertigung Schritt zu halten, wurden zwei P-Scan-Prüfsysteme parallel eingesetzt. Insgesamt wurden 51 Schweißnähte einer P-Scan-Prüfung unterzogen, wobei mit einer dritten P-Scan-Einheit die Auswertung erfolgte.

Die Anforderung des ASME Code, Section XI und der Schweizerischen Behörden verlangen eine volumetrische Prüfung der Mischverbindung der unter Druck stehenden Leitungen. Auch eine Prüfung des Außenoberflächenbereichs ist durchzuführen, was in der Regel mit einer Farbeindringprüfung erfüllt wird. Bei dem Kernkraftwerk Leibstadt lag die Prüfposition zwischen Reaktordruckgefäß und biologischem Schild. Beschränkte Platzverhältnisse und hohe Strahlung erlaubten keine zusätzliche manuelle Farbeindringprüfung, während eine Mechanisierung besonders für das Auffinden von betriebsinduzierten Rissen fragwürdig erschien.

Die Lösung des Problems lag in der Ergänzung der volumetrischen Ultraschallprüfung, um auch die Außenoberfläche zu erfassen. Untersuchungen hatten ergeben, daß dies mit einer flachen Einschallung mit Longitudinalwellen möglich war [11, 12]. Die Prüfung erfolgte dann mit SEL-Prüfköpfen. Die volumetrische Prüfung von Mischverbindungen war das Ziel mehrerer Untersuchungen und internationaler Aktivitäten. Die Herstellung von artgleichen Testkörpern mit Nuten als Referenzreflektoren war immer Voraussetzung.

\section{Komplexe Geometrien, neue Fertigungstechniken und Werkstoffe}

In einer lasergeschweißten Supraleiter-Kabelummantelung für den Fusionsreaktorbau galt es, die Fehlernachweisgrenzen bekannter ZfP-Verfahren im Hinblick auf die bruchmechanischen Erfordernisse aufzuzeigen. Die fabrikationsintegrierte Prüfung mit unmittelbarer Prüfaussage ließ sich mit der positionsgetriggerten hochfrequenten Datenerfassung erreichen (Bild 9) [14]. Die Anzeigen von Nuten und Durchschweißfehlern fallen durch veränderte Muster in den TD-Bildern auf. Die Empfindlichkeit der Methode wurde durch die Kombination der beidseitigen Impuls-Echo-Prüfung und der Durchschallungstechnik verbessert. An entsprechenden Laufzeitsignalen wurde gezeigt, daß durch Verwendung digitaler Signalverarbeitung über den Weg der Merkmalsextraktion und der Mustererkennung die Fehlstellen automatisiert und sicher erkannt werden können.

Mit der Verwendung thermoplastischer Faserverbundwerkstoffe mußten auch entsprechende Prüfverfahren bereitgestellt werden. Bei einer im Jahre 1990 durchgeführten verfahrensvergleichenden Untersuchung an natürlichen Fehlern setzte sich die Ultraschallprüfung durch. Insbesondere bei Fehlern im Bauteilinnern zeigten sich die Vorteile der Ultraschallprüfung im Vergleich zur Thermographie und der Durchstrahlungsprüfung mit der Mikrofokusröhre [14].

Neben den Anforderungen neuer Werkstoffe wurden auch die Formen der zu prüfenden Komponenten und Bauteile schwieriger. Aus den Erfahrungen der Stutzeninnenkanten-Prüfung entsprang die Idee der CAD-unterstützten US-Prüfung. Die bestehende Automation International (AI) Scannermechanik wurde mit einer PC-basierten Steuerung und Datenerfassung erweitert. In Zusammenarbeit mit der Tektrend, Montreal, Kanada, wurde die Soft- und Hardware für die Prüfung rotationssymmetrischer Objekte eingerichtet [15]. Die Leistungsfähigkeit der heute bestehenden Anlage illustriert das Beispiel der Beschichtungsprüfung von Halbkugeln aus Keramik mit 46 bis 56 mm Durchmesser:

Ausgehend von der CAD-Geometrie wird unter Angabe von Einschallwinkel und Abstand des Prüfkopfs zur Prüflingsoberfläche der Steuerungscode generiert. Nach Festlegung des Scanbereichs, einer genauen Positionierung zum Bauteil und der Festlegung der Ultraschall-Prüfparameter kann die Prüfung beginnen. Bei der erwähnten Kugelprüfung wird das Prüfobjekt gedreht, während der Prüfkopf sich äquidistant und senkrecht zur Oberfläche vom Pol zum Äquator bewegt (Bild 10, links). Bild 103, Mitte. zeigt den auf der Basis von Hochfrequenzdaten erstellten Befund einer mit einem Rißnetz durchzogenen Beschichtung mit zwei kleineren Ablösungen. Die Projektion auf die CAD-Bauteiloberfläche veranschaulicht das Resultat (Bild 10, rechts). Die Berechtigung, hier von computerintegrierter Ultraschallprufung zu sprechen, ergibt sich aus der Tatsache, daß alle Schritte von der Prüfvorbereitung bis hin zur
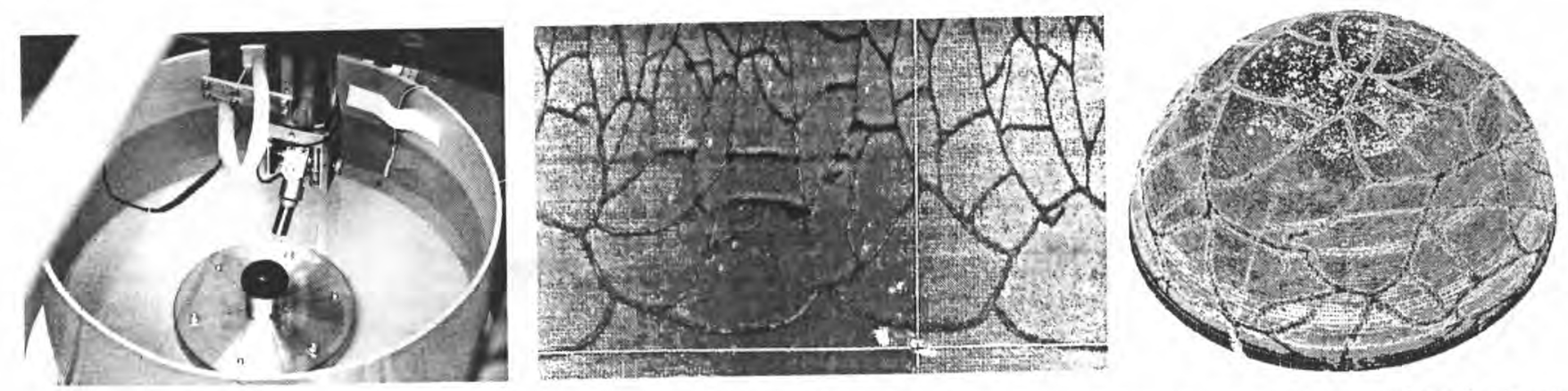

Bild 10. CAD-unterstit-te Beschichtungspriifung von keranischen Halbkugeln: Priifanordnung (links), Befunddarstellung auf der Basis ron Hochfrequenz-Meßdaten (Mitte), C-Scan-Projektion auf die Kugeloberfläche (rechts) 
Dokumentation und Auswertung am gleichen Rechner durchgeführt werden. Mit den Mitteln der digitalen Signalverarbeitung und Mustererkennung ist auch eine automatisierte Auswertung möglich.

\section{Ausblick}

Mit dem vermehrten Einsatz neuer anisotroper Werkstoffe und Werkstoffverbunde wie Kompositen und Beschichtungen steigen die Anforderungen sowohl an die Prüftechniken als auch an die Prüfsysteme. Kürzere Entwicklungszeiten für neue Produkte fordern flexible hochautomatisierte Fertigungs- und Prüfeinrichtungen, Der Schritt zum Einsatz von Prüfrobotern, kombiniert mit einer automatisierten Prüfauswertung, ist absehbar. Aus prüftechnischer Sicht ist anzunehmen, daß die Bedeutung neuer Sende- und Empfangsmethoden zunimmt, z.B. lasererzeugter und interferometrisch empfangener Ultraschall.

\section{Literatur}

1 Testblock mit Hughes-Gerät. Sulzer Prüfattest, Oktober 1946

2 Prüfung einer Versuchsschweißung mit Ultraschall. Sulzer Prüfattest 59104A, August 1953

3 Hornung, R.: Erfahrungen mit der Ultraschallprüfung von Schweißnähten. Konstruktion 14 (1962) 2, S. 41 - 46

4 Edelmann, $X$.; Hornung, R.: Erfahrungen im Prüfen von austenitischen Schweißverbindungen mit Ultraschall. Material und Technik 5 (1977) 1, S. $19-29$

5 Edelmann, X.; Pfister, O.; Hornung, R.: Überlegungen zur Bewertung von Prüfergebnissen bei Ultraschallprüfung von Schweißverbindungen. Materialprüfung 23 (1981) 1, S. 15-21

6 Edelmann,' $X$.: Zur Ultraschallprüfung von Schweißverbindungen. Materialprüfung 28 (1986) 9, S. 262 - 264

7 Edelmann, X.: Wiederkehrende Prüfung mit Ultraschall an austenitischen Schweißverbindungen im Hauptkühlmittelsystem von Druckwasserreaktoren. Internationales Symposium der DGZfP, Saarbrücken 1979, S. 177 - 198

8 Edelmann, X.: Application of Ultrasonic Testing Techniques on Austenitic Welds for Fabrication and In-Service Inspection. NDT International 14 (1981) 3, S.125 - 133
9 Edelmann, X.: Iversen, S.E.: New Developments and Practical Applications of the Ultrasonic P-Scan Technique. 3rd European Conference on Nondestructive Testing, Florenz 1984, Conference Proceedings Volume 1, S. $454-469$

10 Edelmann, $X$.: Voraussetzungen für eine erfolgreiche mechanisierte wiederkehrende Ultraschallprüfung an Rohrleitungen in Kernkraftwerken. DACH Jahrestagung, Lindau 1987, Tagungsband. S. $143-154$

11 Edelmann, X.; Hornung, R.: Investigation of an Ultrasonic Technique for Detection of Surface Flaws During Inservice Inspection of Dissimilar Metal Welds. Fifth International Conference on NDE in the Nuclear Industry, San Diego 1982. Conference Proceedings, S. 180- 183

12 Edelmann, X.: Zehnder, H.; Ulrich J.: Ultrasonic Preservice Inspection at the Austenitic Welds of the Recirculation Piping in the Leibstadt Nuclear Power Plant. 6th International Conference on NDE in the Nuclear Industry, Zürich 1983. ASM Conference Proceedings, S. $239-247$

13 Gribi, M.; Kreier, P.: Mechanisierte Ultraschallprüfung schwierig zu prüfender Werkstoffkonfigurationen und komplexer Geometrie. DGZfP-DACH-Tagung, Luzern 1991. S. 111 - 118

14 Kreier, P.; Gribi, M.: ZfP an neuen Werkstoffen und Fertigungsverfahren mittels digitaler Datenerfassung und Signalverarbeitung. DGZfP-DACH-Tagung, Luzern 1991, S. 63 - 75

15 Kreier P. Gribi M.: Durocher, J.M.: Hay, D.R.; Pelletier, A.; Edelmann. X.: Computer Integrated Testing. European Journal of NDT, 2 (1993) 3 , S. $92-99$

\section{Die Autoren diese Beitrags}

Dipl-Phys. ETH Peter Kreier, geb. 1960, studierte Physik an der ETH Zürich. Seit 1987 ist er Mitarbeiter der Sulzer Innotec AG. Winterthur, Schweiz. Er leitet die Gruppe ZfP der Forschung und Entwicklung des Unternehmens.

Dipl.-Phys. ETH Fernando Allidi, geb. 1953, studierte Physik an der ETH Zürich. Seit 1979 arbeitet er in der Gruppe ZfP im obengenannten Unternehmen

Dipl -Ing René Hornung. Von 1953 bis zu seiner Pensionierung 1988 war er im obengenannten Unternehmen im Bereich der ZfP tätig. Als Leiter der ZfP war er dort maßgeblich an der praxisorientierten Entwicklung und Standardisierung der Ultraschallprüfung beteiligt. Von 1981 bis 1987 war er Präsident der Schweizerischen Gesellschaft für Zerstörungsfreie Prüfung.

Dr. Sc. Techn. ETH Xaver Edelmann studierte Physik an der ETH Zürich. Von 1974 bis 1991 arbeitete er im obengenannten Unternehmen. Er machte sich besonders um die Ultraschallprüfung austenitischer Schweißnähte und der Bestimmung der Fehlergrößen verdient. Heute ist er Direktor der EMPA St. Gallen.

\section{Spektralphotometer: Farbe von Rohstoffen messen}

Die Zeiten, in denen Farbmessung lediglich an Festkörpern praktiziert wurde, gehören ebenso der Vergangenheit an, wie der etablierte Einsatz von Farbmessung lediglich im Rahmen von Endprüfungen. Qualitätsanforderungen werden heutzutage bereits bei der Anlieferung der Rohstoffe (häufig Pulver, Granulate und Schüttgüter) gestellt und ziehen sich wie ein roter Faden durch jeden einzelnen Schritt einer industriellen Fertigung. So aufwendig der "Quality Check" in einzelnen Phasen der Verarbeitung auch sein mag, so sehr zahlt sich die Möglichkeit vorzeitiger Erkennung von Farbabweichungen in barer Münze aus. Mit dem Spektralphotometer Luci 100 der Dr. Lange GmbH, Düsseldorf, wurde der Gedanke der sogenannten ,,präventiven“ Qualitätsprüfung erfolgreich umgesetzt. Der auf Wunsch mitgelieferte Küvettenzusatz beinhaltet eine Küvettenhalterung, mehrere optisch hoch präzise Pulverküvetten sowie Reflexionsstempel und Lichtschutzkappe.

Ein typischer Anwendungsfall der Küvettenmessung liegt bei Baustofflieferanten vor. Hier erweist sich die farbmetrische Bewertung bzw. Differenzierung von z.B. Gips oder Zement mittels verschiedener internationaler Weißgradformen als äußerst hilfreich. In der kunststoffverarbeitenden Industrie hilft die Küvettenmessung bereits Rohprodukte (Pigmente, Granulate) auf Typkonformität hin zu prüfen, ohne selbige erst lösen bzw. aufzuschmelzen und extrudieren zu müssen.

(32446)

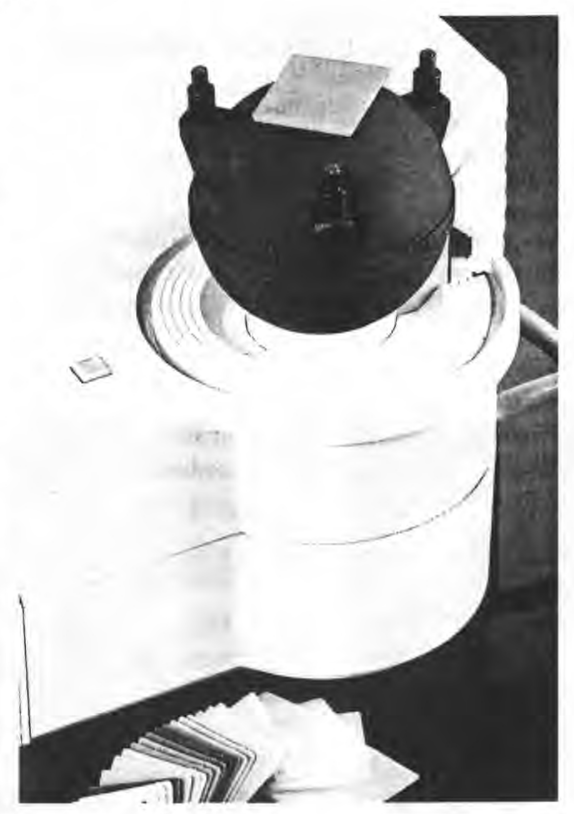

Der flexible Meßßkopf läßßt sich ,. auf den Kopf stellen " und erschütterungsfrei arretieren 